\title{
Gestión del agua residual no doméstica (ARnD) empleando sistema de información geográfica en la definición de distritos sanitarios
}

\section{Non-domestic wastewater management (ARnD) using a geographic information system in the definition of sanitary districts}

\author{
Luis David Arango-Arteaga ${ }^{1}$, Julio César Saldarriaga-Molina ${ }^{2 a}$, \\ Mauricio Andrés Correa-Ochoa ${ }^{2 b}$
}

\begin{abstract}
${ }^{1}$ Profesional de operación negocios, Unidad Gestión de la Información, Agua y Saneamiento, Empresas Públicas de Medellín (EPM). Universidad de Antioquia, Colombia. Correo electrónico: luis.arango5@udea.edu.co. Orcid: 0000-0001-6718-3057

${ }^{2}$ Grupo de investigación GIGA, Facultad de Ingeniería, Universidad de Antioquia, Colombia. Correos electrónicos: ${ }^{a}$ julio.saldarriaga@udea.edu.co, ${ }^{\mathrm{b}}$ mandres.correa@udea.edu.co. Orcid: ${ }^{\mathrm{a}}$ 0000-0002-9395-5417, ${ }^{\mathrm{b}}$ 0000-0003-3666-0767
\end{abstract}

Recibido: 27 octubre, 2020. Aceptado: 12 febrero, 2021. Versión final: 13 mayo, 2021.

\begin{abstract}
Resumen
Gracias al amplio conocimiento que se tiene en las Empresas Públicas de Medellín sobre la problemática de las aguas residuales en el Valle de Aburrá, se procedió a elegir la cuenca La Doctora, localizada en el municipio de Sabaneta, Antioquia, como zona de estudio particular para la aplicación de áreas de presión por vertimientos (carga y volúmenes de caudal). La cuenca se describe como un espacio en el que coexisten características especiales, así: una zona alta (rural), una zona intermedia (combinada entre viviendas actuales y un desarrollo nuevo de estas, más un comercio industrial) y, por último, una zona baja con alta actividad industrial diversa. Con esta investigación se logró diferenciar unas áreas especiales asociadas con componentes de presión por el recurso y por volumen de vertimiento de agua residual, lo que permitió plantear tres diferentes distritos sanitarios: uno para agua residual doméstica (DS-ARD), otro para aguas residuales combinadas (DS ARD-ARnD) y un tercero para aguas residuales no domésticas (DS ARnD). En el estudio se requirió la aplicación de información propia del operador del servicio EPM, herramientas estadísticas, matrices de correlación por caudal y carga contaminante, información de autodeclaración de vertimientos, muestreos de campo y el uso del software ArcGis. Se espera que esta herramienta que tiene amplio componente estadístico pueda ser adoptada por entidades ambientales para mejorar la gestión, planificación y servir de alternativa al tratamiento descentralizado de las aguas residuales.
\end{abstract}

Palabras clave: sistema de información geográfica (SIG); calidad del agua; vertimiento de aguas residuales; clasificación de zonas especiales; gestión del recurso hídrico; mapas de presión-calor.

\footnotetext{
Abstract

Thanks to the extensive knowledge that we have at Empresas Públicas de Medellin, about the wastewater problem $r$ in Aburrá Valley, La Doctora basin located in the municipality of Sabaneta - Antioquia was chosen as a particular study area for the application of pressure areas due to discharges (charge and flow volumes). The basin is described as a

ISSN impreso: 1657 - 4583. ISSN en línea: 2145 - 8456, CC BY-ND 4.0 @) (1) @

Como citar: L. D. Arango-Arteaga, J. C. Saldarriaga-Molina, M. A. Correa-Ochoa, "Gestión del agua residual no doméstica (ARnD) empleando sistema de información geográfica en la definición de distritos sanitarios," Rev. UIS Ing., vol. 20, no. 3, pp. 121-134, 2021, doi: 10.18273/revuin.v20n3-2021008
} 
space in which special characteristics coexist as follows: a high area (rural), an intermediate area (combined between current homes and new development of these, plus an industrial trade), and finally, a low area, with high diverse industrial activity. With this research, it was possible to differentiate special areas associated with components of pressure by the resource and volume of wastewater discharge, which allowed proposing three different sanitary districts. The first one for domestic wastewater (DS-ARD), the second one for water combined wastewater (DS ARD$\mathrm{ARnD}$ ), and the third one, for non-domestic wastewater (DS ARnD). The study required the application of the EPM service operator's information, statistical tools, correlation matrices for flow and pollutant load, self-declaration information for discharges, field sampling, and the use of ArcGIS software. It is expected that this tool, which has a broad statistical component, can be adopted by environmental entities to improve management, planning and serve as an alternative to decentralized treatment of wastewater.

Keywords: Geographic Information System (GIS); water quality; sewage discharge; classification of special zones; water resource management; pressure-heat maps.

\section{Introducción}

La Organización de las Naciones Unidas para la Educación, la Ciencia y la Cultura (UNESCO) presentó el informe mundial sobre el desarrollo de los recursos hídricos "Aguas residuales: el recurso desaprovechado", que concuerda con los contenidos de La Agenda 2030 para el Desarrollo Sostenible [1]. En este sentido, la organización exhibió entre sus consideraciones las consecuencias del vertido de las aguas residuales (AR) no tratadas o con tratamiento inadecuado y su efecto en las municipalidades. Igualmente, habla sobre el reto que encarna la gestión de dichas aguas para las instituciones y expone la "brecha en materia de conocimiento, investigación, tecnología y creación de capacidades" y la necesidad de un marco jurídico coherente y comprometido. En este sentido, el informe hace énfasis sobre la amplia brecha en el conocimiento, la tecnología aplicada, la investigación realizada y las oportunidades generadas en materia de gestión del recurso hídrico y en específico de las AR. Es importante mencionar que no se ha logrado encontrar publicaciones o trabajos orientados hacia la descentralización en el tratamiento parcial del agua residual en zonas o cuencas con convergencias de población e industria con baja planeación, por lo que se presentarán más adelante algunos aportes que orientan la necesidad de pensar en cómo mejorar el trabajo futuro.

Algunos trabajos e investigaciones aportan resultados a la actual situación mundial, en relación con la calidad del agua. Dentro de ello, se logra observar cómo aspectos relacionados con la presión por el recurso, el aumento de la población y los vertimientos no controlados a fuentes de agua se pueden convertir en detonantes que implican la toma de decisiones y la búsqueda cada vez más acelerada de políticas de uso, manejo y control del vertido de residuos líquidos para el sector.

Asegura [2] que los asentamientos humanos han propiciado la contaminación de las fuentes de agua y han creado una presión por adoptar las respuestas institucionales locales y las tecnologías de abastecimiento de agua y de redes de alcantarillado, desde mediados del siglo XIX. De allí que para finales del mismo siglo muchos estados hayan propiciado la aceptación de políticas frente a la contaminación y que estas permitan proteger el suministro público de agua de la contaminación por aguas residuales. A continuación, se presentan algunas investigaciones cuyo enfoque está orientado a la protección y uso del agua, debidos entre otros a problemas de escases y necesidad de garantizar el uso futuro del agua y las estrategias que se presentan en algunas regiones hacia una mejor gestión del recurso.

Se quiere destacar la búsqueda que hace el ser humano por alcanzar un desarrollo tecnológico que garantice la seguridad alimentaria frente a una explosión demográfica cada vez mayor, nuevas industrias y servicios, que terminan con grandes impactos sobre el recurso agua y que alcanza valores no observados antes, lo cual se traduce en mayores volúmenes de agua de calidad compleja, donde su tratamiento genera retos para las comunidades. De acá que la calidad del agua sea vista como una amenaza para la salud de las personas, con la aparición de enfermedades emergentes y reemergentes [3].

Para [4] un ejemplo que permite explicar lo anterior se observa en la India, donde la rápida industrialización, la urbanización y la expansión de la población implican mayor demanda por recurso, lo que ha creado una serie de problemáticas ambientales, donde la contaminación hídrica se convierte en la mayor afectación, tanto en su calidad como en la cantidad para fuentes superficiales y subterráneas; todo ello redunda posteriormente en la disponibilidad, pues no se cuenta con vacíos en su estructura existente para garantizar agua potable y controlar sus vertimientos en las redes y cuerpos de agua, para hacer más sostenible dicho recurso. 
Algunos trabajos novedosos frente al manejo del agua incluyen lo reportado por [5]. Estos investigadores indican cómo diferentes empresas prestadoras de servicio buscan cambiar deliberadamente la infraestructura para servir el agua y el alcantarillado, ello orientado hacia la mejora en la sostenibilidad de la prestación dentro de un marco comparativamente regulado, y valorando la capacidad de innovar con procesos de inversión de capital y resultados de desempeño.

También se podría incluir lo ilustrado por [6], donde en Turquía, quien, dada su escasa infraestructura ambiental y su posibilidad de ingresar a la Comunidad Económica Europea, implementa un modelo de gestión integrada de cuenca hidrográfica (conocida como IEBM), cuyo propósito es administrar mejor sus recursos y superar, así, la degradación y contaminación de los recursos naturales, garantizando un mejor manejo del agua. Esta herramienta corresponde a una nueva mirada administrativa con aplicación internacional, como la Directiva Marco de Agua de la Comisión Europea.

Otros autores [7] hacen énfasis en que la definición operativa de la calidad del agua debe estar motivada por la discusión de métodos efectivos, pero sencillos de tratamiento de aguas residuales. Estos investigadores llaman la atención en lo complejo que es la toma manual de las mediciones de muestreo y la limitación inherente del análisis de los datos de teledetección en muchos sitios. A este respecto, [8] ha realizado estudios como el de mediciones de línea base para la concentración de nutrientes y las propiedades fisicoquímicas del agua en zonas costeras, lo que permite, por ejemplo, entender la variación espacial de compuestos como los nutrientes y el patrón fisicoquímico del agua en la costa de Port Dickson [8].

Se destaca, también, un enfoque que involucra los indicadores de calidad del agua y que puede ser utilizado para caracterizar el estado del recurso, cuantificar y calificar el cambio de los ecosistemas bajo diferentes regímenes de perturbación. En este, [9] indica que los modelos de calidad del agua, basados en procesos, pueden predecir las variaciones espaciotemporales de los indicadores de calidad y proporcionar información útil para los responsables de la formulación de políticas sobre la gestión racional de los recursos hídricos.

Ahora, en cuanto al ámbito colombiano, el país no es ajeno a la tendencia global en el manejo del recurso hídrico y gradualmente ha venido implementando leyes y regulaciones ambientales y políticas públicas, que buscan proteger la salud humana desde la conservación del agua, mitigando el impacto de las poblaciones sobre los recursos naturales [10]. Igualmente, están algunas normas nacionales como la Resolución 0075 [11] y el Decreto 2667 [12], que señalan la exigencia de monitorear las cargas contaminantes registradas por los usuarios, los datos prioritarios para el reporte a la autoridad ambiental competente y para el cálculo de cargas contaminantes y tasas retributivas.

En el país se presenta la Resolución 0631 [13], que, además de clasificar las aguas residuales domesticas (ARD) de las no domésticas (ARnD), establece valores límites máximos permisibles para vertimiento y crea 8 sectores y 73 actividades específicas. Además, abre una oportunidad de diagnosticar e integrar la información de la calidad del agua en las redes de alcantarillado, a partir de conocer y registrar hasta 57 parámetros contaminantes por vertimiento de agua residual no doméstica (ARnD) en el sitio de descarga; variables que son ajustadas según la actividad productiva del usuario del sistema de alcantarillado público.

De otro lado, la Resolución 0631 de 2015 introduce cambios importantes en la forma de gestionar los vertimientos de ARnD y da paso al control de contaminantes por concentración, estableciendo límites máximos permisibles, de acuerdo con los parámetros químicos presentes y conforme al sector productivo en el cual se encuentre clasificada la industria usuaria del alcantarillado. Este control debe ser realizado por las empresas de servicios públicos domiciliarios y exige a los usuarios generadores de ARnD los respectivos estudios de caracterización de vertimientos.

Finalmente, la legislación colombiana, liderada por [13] y [14], establece los parámetros y valores máximos permisibles en los vertimientos a cuerpos de agua superficiales y a los sistemas de alcantarillado público, lo que se configura como el máximo desarrollo del marco legal nacional y se convierte en el punto de partida de la presente investigación. Con el presente trabajo se busca evaluar el impacto que generan las diferentes fuentes de vertimiento en la cuenca La Doctora del municipio de Sabaneta (una cuenca de altas pendientes y que cuenta con una zona rural definida, un sector mixto de población y comercio y, en la parte baja, una industrial diversa y de gran volumen), en la que el alcantarillado recibe la diversidad alta de vertidos, a partir de sus actividades económicas reconocidas y los usos del suelo evidenciados (fabricación y manufactura de bienes, alimentos y bebidas, agroindustria y ganadería, servicios y otras actividades), para, finalmente, definir los denominados distritos sanitarios. 
En la figura 1 se localiza el sitio de estudio, el cual está enmarcado dentro del municipio de Sabaneta, Antioquia, a $14 \mathrm{~km}$ al sur del municipio de Medellín, a una altura de $1578 \mathrm{~m} \mathrm{~s}$. n. m. y con una temperatura promedio de $21^{\circ} \mathrm{C}$.

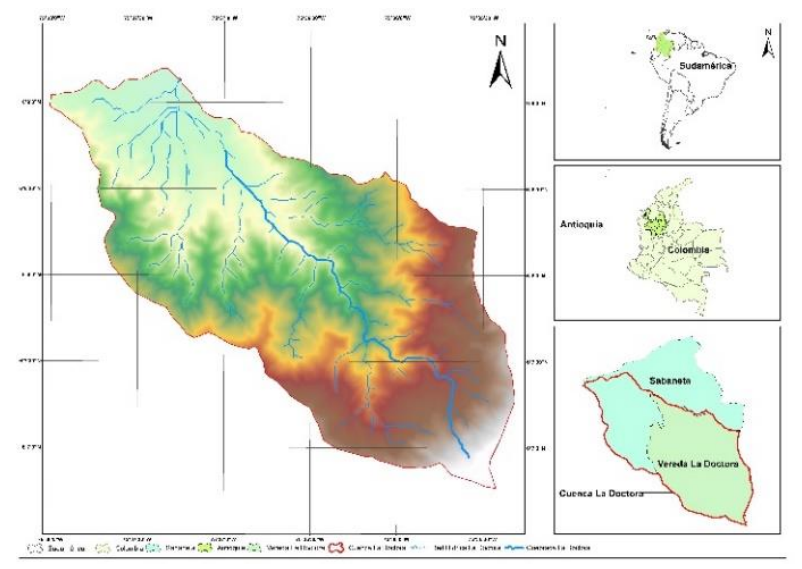

Figura 1. Localización en Colombia y Antioquia de la zona de estudio (cuenca La Doctora-Sabaneta).

\section{Metodología}

Para lograr la definición de los distritos sanitarios en la cuenca La Doctora, se describen, a continuación, todas las fases propuestas. Para el desarrollo de la investigación, fue clave el uso de sistemas de información geográfico (SIG), con el cual se logró establecer una línea base sobre la dinámica de los vertimientos transportados por la red de alcantarillado en la cuenca, que posteriormente son conducidos a la Planta de Tratamiento de Agua Residual (PTAR) San Fernando (Operada por EPM). Algunas de estas actividades son expuestas a continuación.

\subsection{Determinación del caudal de vertimientos de la cuenca La Doctora y de la calidad de los vertimientos}

Para el caudal de vertimiento se emplearon los registros de facturación de los usuarios de alcantarillado activos en la cuenca La Doctora (información propia de EPM) y se usó la herramienta ArcGis [15] en los análisis geoestadísticos. Adicionalmente, para conocer la calidad de los vertimientos en la cuenca, se hizo una determinación específica, en esta se consideraron 57 parámetros contaminantes del AR (información EPM), teniendo en cuenta la normatividad colombiana [13]. También se obtuvo información a partir de las bases de datos generadas como resultado del trabajo de la unidad de monitoreo y control de aguas residuales de EPM y los registros de las autodeclaraciones de los usuarios con vocación industrial de la cuenca (información propia del operador EPM). Estas autodeclaraciones corresponden a estudios de caracterización de AR de los procesos industriales de usuarios generadores de $\mathrm{ARnD}$ y con estas se obtuvo el panorama del estado y los potenciales riesgos de dichos vertimientos a la red de alcantarillado público.

\subsection{Comprobación de datos, cálculo de agregaciones y potencialidad de programas de monitoreo control de vertimientos}

En el desarrollo de la investigación se empleó estadística tradicional (herramienta R Studio) y otros análisis como matriz de correlación por concentración de $\mathrm{ARnD}$, matriz de correlación por caudal, matriz de correlación por carga contaminante y la prueba de normalidad de datos. También se realizó un análisis de agregaciones espaciales en el que se usaron métodos geográficos novedosos, como la aplicación del software ArcGis. Se destaca la agregación espacial de autodeclaraciones por caudal, por carga contaminante y por color. Por último, se empleó el método de Kernel y se propuso rasterizar los análisis de distribución espacial usando imágenes como píxeles, de acuerdo con su distancia euclidiana y en función de su caudal, carga contaminante o concentración, según el caso.

\subsection{Determinación de los distritos sanitarios (DS) y de la calidad del AR del colector La Doctora}

Para el cálculo o la determinación de los distritos de AR se hizo un análisis empleando el software ArcGis. En este análisis se clasificaron tres diferentes distritos sanitarios: DS asociado directamente con descargas de aguas residuales, DS del sector mixto (aguas residuales domésticas y no domésticas) y, por último, los DS donde solo se reciben aportes de vertimientos relacionados con ARnD. En cada distrito se contó con un total de cinco muestras colectadas por un laboratorio especializado (Laboratorio Microbiológico Ortíz -Martínez) y teniendo en cuenta las normas contenidas en el Estándar Métodos, 2019 [16], distribuidas entre septiembre de 2018 y marzo de 2019, buscando incluir aspectos asociados con la estacionalidad (invierno, verano y transiciones).

De otro lado, en el cumplimiento del objetivo de determinación de la calidad del AR del colector, se realizaron muestreos en campo, ajustados a estándares del Instituto de Hidrología, Meteorología y Estudios Ambientales (IDEAM) [17]. Todas las muestras se colectaron, almacenaron y preservaron, de acuerdo con lo establecido en el protocolo para el monitoreo del agua establecido en [17]. En total, se seleccionaron tres puntos de muestreo en la cuenca (parte alta, centro y baja), con cinco muestreos distribuidos en las zonas definidas de la cuenca; sus resultados se encuentran en un documento no 
público, que hace parte del material de soporte que entrega el laboratorio a EPM y a esta se le dio acceso para complementar los alcances de la presente investigación.

Por último, después de obtener los resultados individuales, así como la información de caudal, cargas contaminantes, daño potencial y color, se realizó la integración de las áreas de densidad de cada componente y se calculó esta área, a partir de una suma ponderada. Es decir, se determinaron las áreas de presión-calor, resultantes del análisis integrado de información, a partir de la operación de álgebra de mapas. Con esta información, se procedió a elaborar mapas de calor presión de los vertimientos de AR en la cuenca, empleando, también, información de autodeclaraciones de vertimientos a la red (información que se ha indicado, fue soportada por EPM).

\section{Resultados}

\subsection{Vertimientos de agua residual}

Apoyados en la resolución 0631 de 2016 [13], se realizó la reclasificación de los usuarios del servicio de alcantarillado en la zona de estudio. Con base en dicha reclasificación y con el apoyo en los registros de facturación de EPM, se encontró que se vierten al alcantarillado 491.828,66 $\mathrm{m}^{3}$ promedio mes, que corresponden al caudal de AR de la población, en función del volumen mensual de vertimientos. También se tiene que, a partir de la información procesada en la investigación, fue posible establecer que el $75 \%$ de la población (un total de 21.942 puntos de vertimiento de ARD) aporta un caudal promedio menor a $14,38 \mathrm{~m}^{3} / \mathrm{mes}$. Este total de vertidos corresponde al $50 \%$ de ARD descargadas, correspondientes a $179.343 \mathrm{~m}^{3} / \mathrm{mes}$.

En términos de ARnD, se encuentra que un $25 \%$ de estos vertimientos están asociados a los más altos caudales, lo que implica variaciones importantes en la dinámica de la cuenca. Por lo tanto, dichos vertidos equivalen a 728 puntos y, a su vez, explican el $91 \%$ del ARnD descargada en el municipio de Sabaneta.

Como se puede observar en la figura 2, se registran para la zona de estudio los sitios o puntos de vertimiento en la cuenca La Doctora; dicha información está referida según las clasificaciones específicas: los puntos rojos y amarillos, los de menor aporte (inferiores a $\left.19,23 \mathrm{~m}^{3} / \mathrm{mes}\right)$; verdes y azules, las mayores descargas (hasta $8.568 \mathrm{~m}^{3} / \mathrm{mes}$ ). Es decir, los puntos verdes $\mathrm{y}$ azules, relacionados con las mayores descargas de ARnD, corresponden a un $50 \%$ de los aportes y significan un total de 1.165 descargas $\left(67.689,075 \mathrm{~m}^{3} / \mathrm{mes}\right)$.
De otro lado, llama la atención que esta población tan significativa se agrupa en áreas geográficas muy definidas en la cuenca (partes media y baja).

En la parte media de la cuenca se registran fenómenos de expansión urbana y se encuentran industrias de sectores de la construcción, así como otras asociadas al desarrollo de vivienda. Conjuntamente, en la parte baja de la figura 1 (zonas nororiental y noroccidental del mapa) se encuentran parques y bodegas especializadas, relacionadas con actividades industriales, que en su mayoría representan sectores de manufactura de bienes y procesamiento de alimentos y bebidas.

La tendencia de agrupación en áreas geográficamente definidas ayuda a dar validez a la hipótesis planteada en la investigación, pues permite seleccionar áreas o distritos diferenciales de $\mathrm{AR}$ al interior de las cuencas hídricas, de acuerdo con la clasificación de los vertimientos puntuales al alcantarillado público.



Figura 2. Distribución espacial de frecuencias ARnD en la cuenca La Doctora. Fuente: elaboración propia.

\subsection{Análisis de vertimientos por calidad de ARnD}

Como se observa en la figura 3 , en total se evaluaron 48 puntos de vertimiento industrial en la cuenca. Los puntos se seleccionaron a partir de la información propia de la base de datos de autodeclaraciones de EPM (estudios de caracterización de AR), clasificados por [14] en cinco 
actividades productivas; se incluyen sectores como: fabricación y manufactura de bienes, alimentos y bebidas, agroindustria y ganadería, servicios y otras actividades, entre otros.

Dado que el total de usuarios que componen el sistema de alcantarillado corresponde a 728 puntos, se puede deducir que los 48 puntos corresponden a una muestra del $6,6 \%$ de la población y que arrojaron un caudal total de $686.887 \mathrm{~m}^{3} / \mathrm{mes}$ (información cotejada con la base de datos de autodeclaraciones de EPM).

De acuerdo con los puntos de vertimiento de $\mathrm{ARnD}$ incluidos para el estudio, se identificó que el sector de fabricación y manufactura de bienes es el primer aportante de caudal contaminante al alcantarillado, con un total declarado de $551.179 \mathrm{~m}^{3} / \mathrm{mes}$, que representan cerca del $80 \%$ del total de aporte según los reportes de autodeclaraciones.

El segundo sector aportante corresponde a producción de alimentos y bebidas, con un 1,5\% del total del registro $\left(8.677 \mathrm{~m}^{3} / \mathrm{mes}\right)$. Además, según el sistema de facturación de EPM, el ARnD alcanza un vertimiento de $135.378,15 \mathrm{~m}^{3} / \mathrm{mes}$, donde el aporte de los dos sectores descritos hace que el volumen de vertimientos declarado esté en un $414 \%$ por encima de los valores totales registrados, según el sistema de micro medición. Este valor del caudal obtenido por autodeclaraciones muestra un desfase importante entre el registrado por micromedición y el tomado en las autodeclaraciones, y para efectos del estudio se tomaron los datos obtenidos por la micromedición para la estimación de caudales puntuales.
Es importante mencionar que, aunque las actividades de agroindustria y ganadería en la cuenca son pocas, estas generan altas cargas contaminantes, alcanzando una carga promedio de $45.000 \mathrm{~kg} / \mathrm{mes}$ en términos de demanda química de oxígeno (DQO).

\subsection{Análisis de correlaciones}

De otro lado, a partir del análisis de matrices, se logró agrupar los parámetros contaminantes provenientes de las autodeclaraciones de los usuarios industriales que corresponden con los exigidos en el Decreto 1076 de 2015 [14], según su concentración y en función del tipo de actividad productiva [13]. Del análisis, se identifica la correlación entre las actividades productivas de las industrias y los parámetros contaminantes, así como la relación entre los contaminantes presentes en los muestreos de AR para los puntos seleccionados.

Con base en lo anterior y como resultado de las correlaciones, se encontró que existe una alta respuesta entre los parámetros cloruros y nitrógeno con el color y los valores de $\mathrm{pH}$ máximo, que alcanzan valores de 0,99, e inversamente proporcionales con los valores de $\mathrm{pH}$ mínimo, que registran datos cercanos a cero. Estas respuestas de correlación se presentan gracias a la presencia en la cuenca de industrias y sectores de manufactura, dedicadas a procesos de tinte, estampación y lavado de textiles, que tienen vertimientos con valores de $\mathrm{pH}$ superiores a las nueve unidades, y en los que el proceso textil incorpora iones y detergentes al AR.

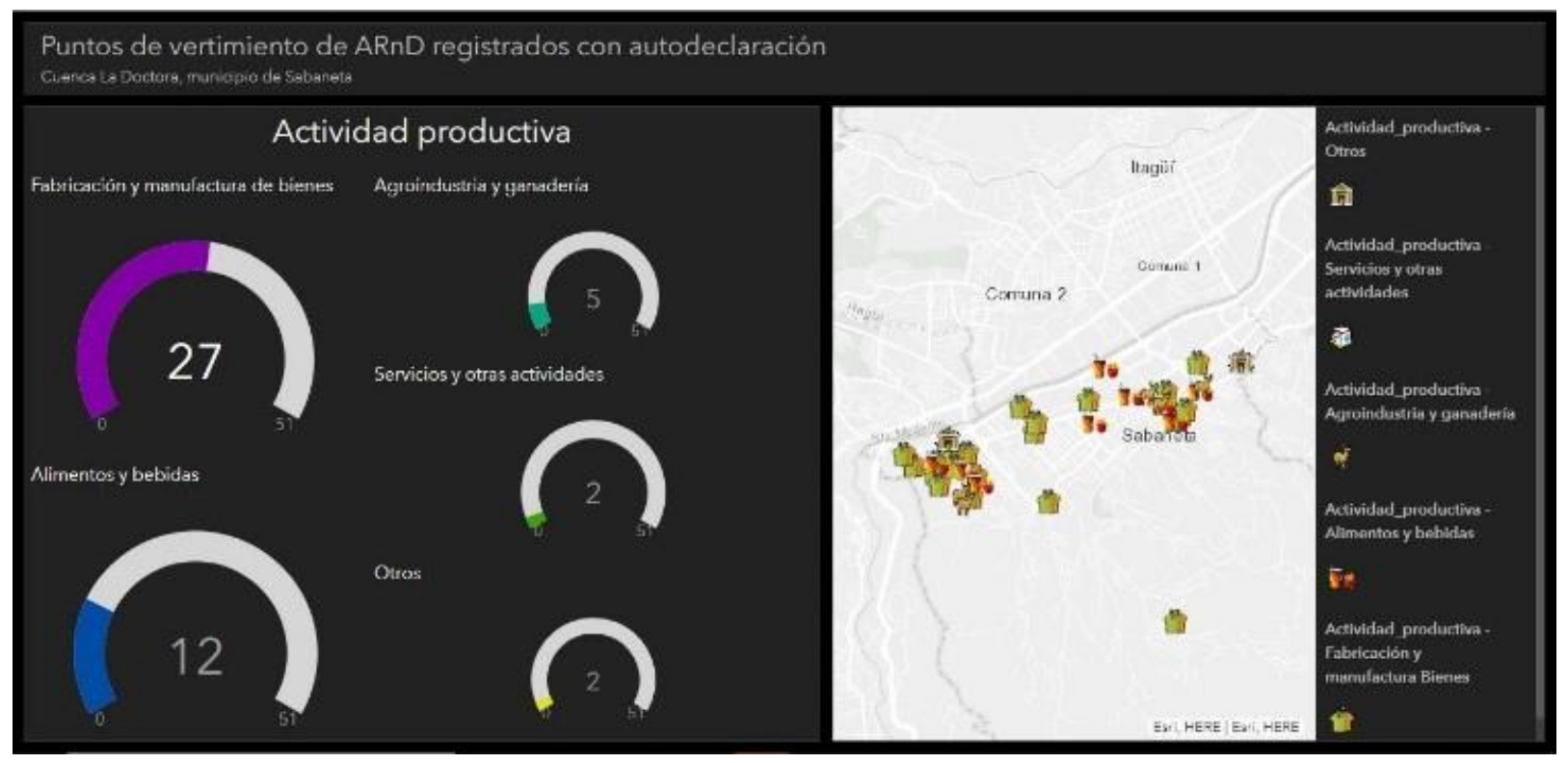

Figura 3. Distribución espacial de frecuencias ARnD en la cuenca La Doctora. Fuente: elaboración propia 
Adicionalmente, se observa que el tipo de actividad productiva no presenta correlaciones directas con ningún contaminante de las autodeclaraciones obtenidas, pues el mayor valor registrado es para el $\mathrm{pH}$, con apenas 0,32 .

De este análisis se hacen dos consideraciones importantes: una es un resultado relevante, dado que el color, al ser un contaminante de alto impacto en las fuentes hídricas, deberá ser monitoreado constantemente en la red, evitando afectaciones ambientales importantes; dos, para lograr la medición constante de esta variable, se deberá estimar la posible ubicación de equipos de medición (sensores), que permitan la cuantificación en tiempo real y la toma de decisiones por parte de la autoridad ambiental competente.

\subsection{Prueba de normalidad de los datos y estimación de la densidad de Kernel}

Para los datos calculados en la investigación y tras emplear herramientas estadísticas tal como ocurrió con la prueba Shapiro Wilk, que permitió conocer valores $\mathrm{p}$ inferiores a 0,05 y deducir que los datos no presentan normalidad, no se pueden aplicar métodos estadísticos paramétricos y, por ello, se procede a comparar los datos de forma pareada.

De lo anterior, se procedió a aplicar el análisis de comparación espacial mediante la densidad Kernel, y se determinaron áreas de presión por contaminantes en la cuenca La Doctora. Igualmente, a partir de los análisis preliminares de distribuciones espaciales se realizaron análisis estadísticos que permitieron determinar el tipo de agregación o dispersión que presentan los datos, y, con ello, se pudo obtener el patrón de distribución de los valores de vertimiento.

En consecuencia, se analizaron tres capas de información, de acuerdo con las 48 autodeclaraciones seleccionadas para la cuenca y de acuerdo con las siguientes agrupaciones:

\subsubsection{Daño potencial a la red (pH y grasas y aceites)}

Este concepto se introduce a partir de los parámetros contaminantes que, de acuerdo con características fisicoquímicas, puede ocasionar daños en la red de alcantarillado, ya sea por obstrucciones debidas a presencia de grasas y aceites o por daños estructurales a las tuberías, derivados de vertimientos con $\mathrm{pH}$ ácidos o básicos.

En la figura 4 se presenta un detalle de la información relacionada con el daño potencial a la red, obtenido a partir del uso de ArcGis y de la información por autodeclaraciones. En dicha figura se resumen los daños potenciales a la red destacados, con base en los puntos críticos, donde se relaciona la variable $\mathrm{pH}$ a partir de colores oscuros, mientras que las altas cargas contaminantes (parámetro grasas y aceites) son representadas a partir del tamaño del círculo. Por lo tanto, mientras más oscuro sea el punto, se presenta un valor más crítico de $\mathrm{pH}$ para la red de alcantarillado, y, de otro lado, su tamaño se asocia con la concentración de grasas $\mathrm{y}$ aceites presentes en el vertimiento.

Como segundo elemento, se evidencia la presencia de un punto de vertimiento con una alta concentración (3.559 mg/L) para grasas y aceites, en comparación con la media de la muestra (38 mg/L). Igualmente, se ubican puntos en zonas industriales que sobrepasan los valores permitidos de $\mathrm{pH}$ y que son potencialmente destructivos para la red, con máximos de hasta 12 unidades en vertimientos básicos y hasta de 2,81 unidades en vertimientos ácidos.

\subsubsection{Cargas contaminantes (DQO, DBO, SST)}

Como se muestra en la figura 5, en azul oscuro y según el tamaño, se identifican los puntos de vertimiento con altas cargas contaminantes y relación DBO/DQO. Existen puntos con altas cargas que están asociados con variables como la demanda química de oxígeno (DQO), la demanda bioquímica de oxígeno (DBO) y los sólidos suspendidos totales (SST). También, se encontró que las relaciones alcanzan valores de hasta 0,52 (con máximos y mínimos que oscilan desde 0,1 hasta 14 ton/mes en DBO y entre 0,1 hasta 30 ton/mes para DQO), y la carga contaminante por SST se representa con el tamaño de los círculos, donde las cargas de sólidos alcanzan hasta 28 ton $/$ mes.

Para la variable color, se muestran en la figura 6 los puntos de vertimiento con las mayores respuestas de la variable (color real en la cuenca), donde el tamaño de los círculos es asociado con alto nivel de aporte. El máximo obtenido fue de 246 unidades de color real (leído a $436 \mathrm{~nm}$ de longitud de onda).

\subsection{Mapa de calor-presión por vertimientos en la cuenca La Doctora (Kernel)}

A partir de la superposición de los mapas de calor obtenidos para ARD y ARnD, se encontraron algunos resultados que serán analizados en el apartado de discusión de resultados. Sin embargo, para evaluar la presión que soporta para la zona de estudio los vertimientos, se construyó la figura 7 , donde el azul con aspecto difuso representa las zonas de aporte de ARD y el amarillo, rojo y naranja, aquellas que registran mayor presión contaminante por ARnD. 


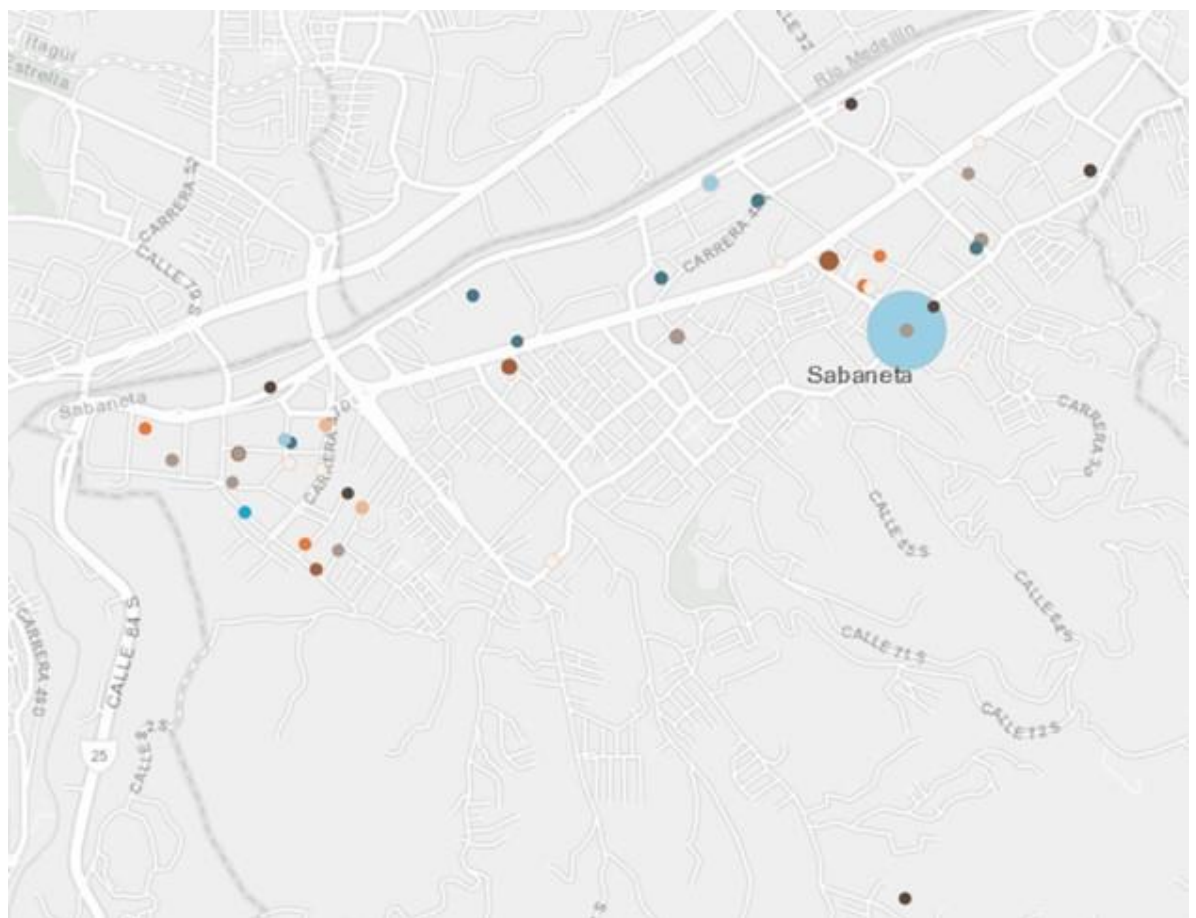

Figura 4. Autodeclaraciones por daño potencial a la red. Fuente: elaboración propia.



Figura 5. Autodeclaraciones de acuerdo con carga contaminante. Fuente: elaboración propia. 


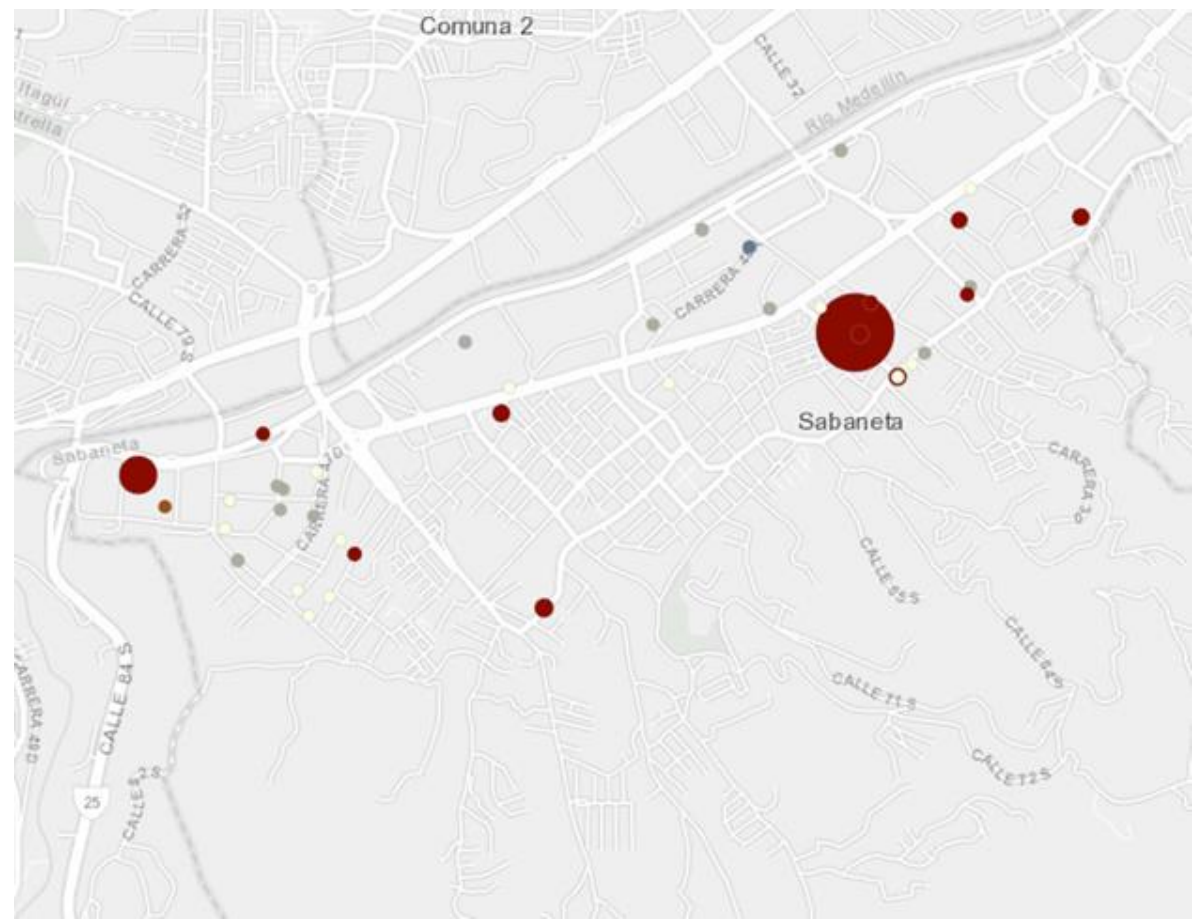

Figura 6. Autodeclaraciones según la variable color real. Fuente: elaboración propia.

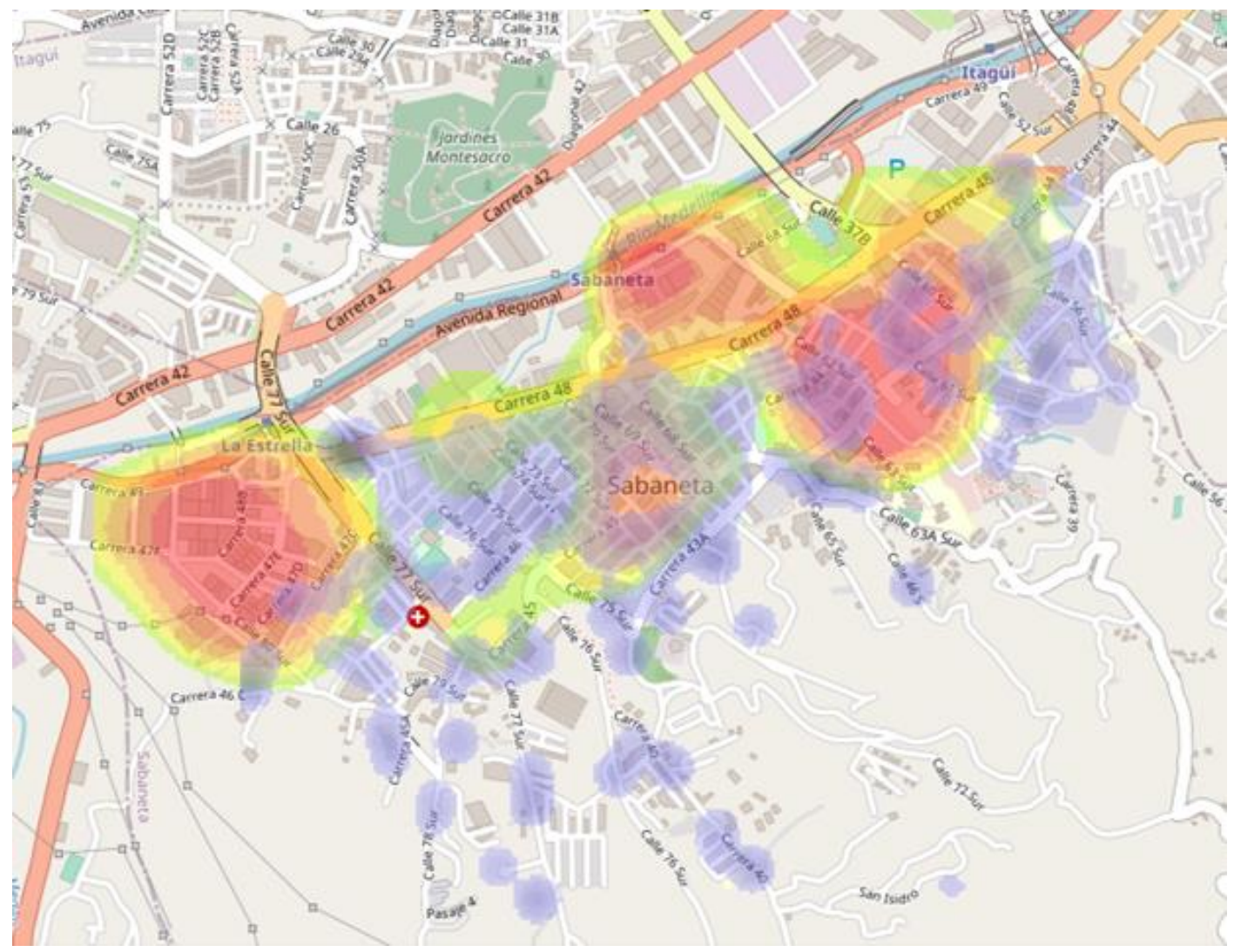

Figura 7. Mapa de calor-presión de los vertimientos de agua residual. Fuente: elaboración propia. 
De la figura se puede observar que en la parte central se registra un fenómeno de alta concentración de vertimientos en el área, relacionado con el sector de la construcción, pues la zona tiende a ser llamativa para el uso residencial. De acuerdo con los resultados obtenidos se encuentra que el ARnD presenta un comportamiento espacial agregado, según el caudal promedio mensual entregado a las redes de alcantarillado público $\left(66.886 \mathrm{~m}^{3} / \mathrm{mes}\right)$. Los resultados permitieron observar que la carga contaminante está definida según el caudal y el tamaño de la industria, y no con base en el tipo de actividad productiva [14].

Es de destacar que no fue posible encontrar correlación directa entre el tipo de industria clasificada, según la normatividad colombiana, y los niveles de carga contaminante presentes en los vertimientos (expresados en términos DQO, DBO y SST). Con lo cual se puede inferir que en la cuenca los niveles contaminantes de carga (ton/mes) no pueden determinarse de acuerdo con la clasificación dada por la norma [13], y se comprobó un patrón de agrupamiento de los datos que permitió identificar zonas diferenciales y agrupamientos significativos en el área de estudio, todo en función del caudal aportado a las redes de alcantarillado. Lo anterior permite responder a la hipótesis de partida e indica que en la cuenca se generan zonas diferenciales de presión, según el volumen de vertimiento agrupado. Igualmente, se registran áreas al interior de la cuenca, donde se concentran volúmenes altos de vertimientos de $\mathrm{AR}$, de dos tipos: diferencial ARD y no diferencial ARnD.

Con todos estos resultados, se pudo identificar el patrón de agregación espacial debido a los vertimientos, que permitirá, a su vez, orientar el establecimiento de las áreas diferenciadas al interior de la cuenca y que permite definir los denominados distritos sanitarios.

\subsection{Delimitación de distritos sanitarios (DS)}

A partir del planteamiento anterior, se construyó una delimitación de áreas (ver figura 8), y con ellas se establecieron tres diferentes distritos sanitarios: en amarillo se presenta el DS asociado con ARD; en azul, el DS mixto; y en magenta, el DS exclusivo para ARnD. Estos tres distritos son definidos a continuación.

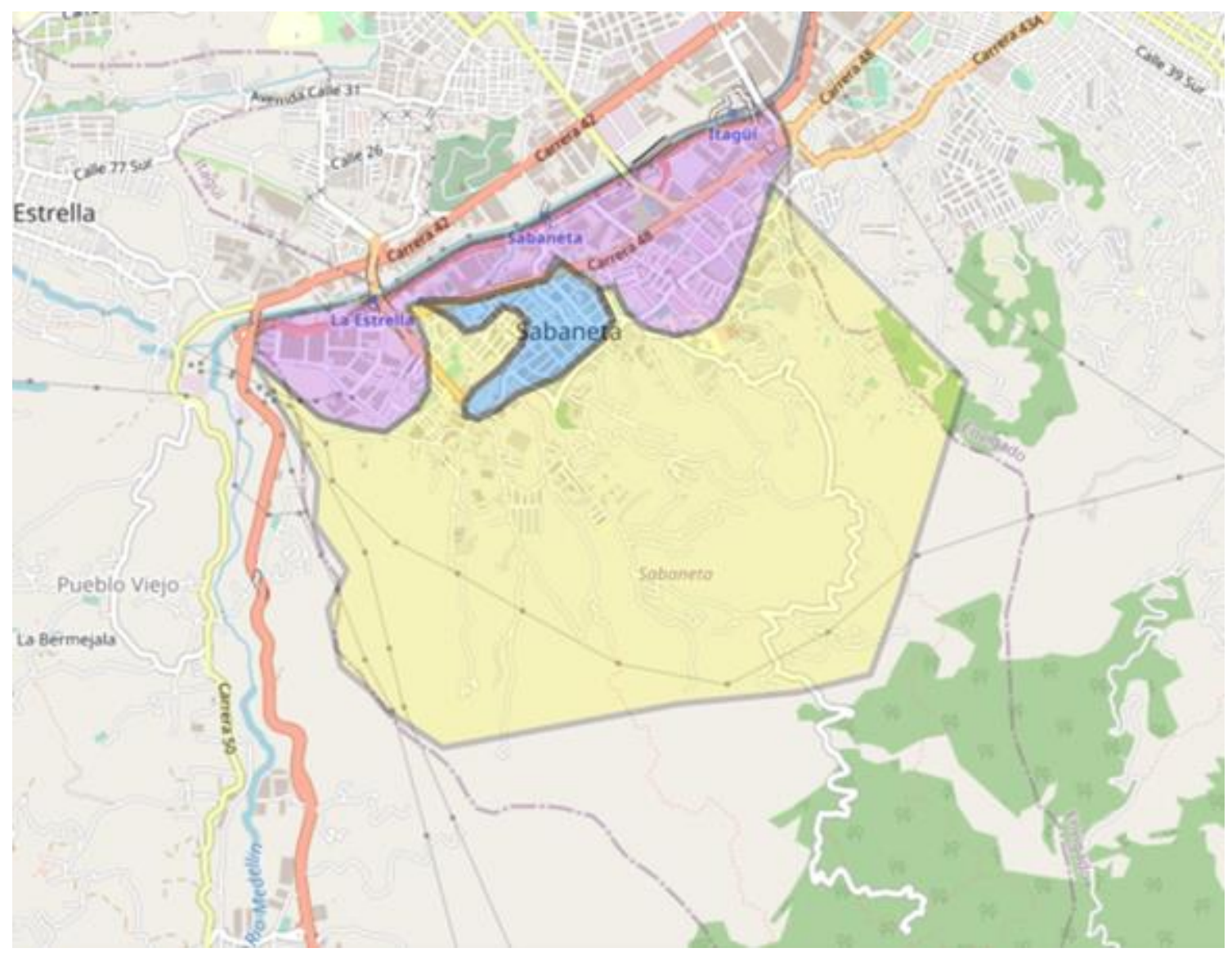

Figura 8. Establecimiento de distritos sanitarios en la cuenca La Doctora. Fuente: elaboración propia. 


\subsubsection{Distrito sanitario de agua residual doméstica}

En la figura 8 (color amarillo) se observa el distrito sanitario de agua residual doméstica (DS ARD), este distrito cubre un área estimada de $7,76 \mathrm{~km}^{2}$ y se observan fenómenos de expansión urbana en sitios que cambian su uso del suelo y que tradicionalmente se definían como áreas semirurales. Este fenómeno ha implicado el asentamiento de importantes proyectos de construcción de vivienda, que vienen incrementado el volumen de vertimientos transportados por las redes de alcantarillado instaladas en dichas áreas.

\subsubsection{Distrito sanitario mixto (DSM) o de aporte de ARD y ARnD}

Corresponde a la zona central del municipio de Sabaneta y reporta un fenómeno de interés para el control de vertimientos. Lo anterior, debido a que existe una alta presión de ARnD por caudal, a pesar de no contar con registros de autodeclaraciones de industrias en la zona. Esta parte de la cuenca registra una alta presión de ARD $\mathrm{y}$, por lo tanto, es un distrito sanitario (véase figura 8 , color azul) que alcanza un área cercana a los $0,4 \mathrm{~km}^{2}$.

\subsubsection{Distrito sanitario de agua residual no doméstica (DS ARnD)}

El último DS definido y que corresponde exclusivamente a una zona con alta presión por vertimientos de ARnD es identificado en la figura 8 (color magenta). Esta zona ocupa un área total en $2,3 \mathrm{~km}^{2}$, y en ella se registran aportes de parques industriales, ubicados dentro de su área de influencia.

\subsection{Variabilidad de la calidad del agua residual en la cuenca}

De acuerdo con el análisis de la información, se pudo establecer que gracias a la combinación de herramientas como el ArcGis y las bases de datos con que cuenta el prestador del servicio de alcantarillado en la zona de estudio (EPM), es posible el establecimiento de las zonas definidas en el apartado anterior.

Se logró establecer que el colector La Doctora tiene una longitud de 4.189 metros, compuestos por 140 tramos de tubería de alcantarillado, y recibe los vertimientos de AR desde la parte alta de la cuenca (zona semirural), pasando por la parte media y baja, conocidas como zonas urbanas. En este sistema de alcantarillado son recogidas las aguas que componen los diferentes DS.
Como se ha indicado, se ubicaron tres puntos de muestreo específicos: parte alta, parte media y zona baja; puntos que coincidieron con ubicaciones especiales dentro de los distritos resultantes. En los muestreos se llevaron a cabo mediciones de caudal y cuantificación de las variables correspondientes en las diferentes muestras (DQBO, DBO, sólidos, nitrógeno total, grasas y aceites, cloruros, color, $\mathrm{pH}$, conductividad, entre otros), que fueron evaluadas (toma, custodia y análisis) por un laboratorio externo especializado, garantizando el cumplimiento de las normas y técnicas definidas por la entidad ambiental competente [16].

En cada punto se realizaron estudios de calidad durante cinco meses (septiembre de 2018 a febrero de 2019), y en total se realizaron cinco muestreos por sitio. De acuerdo con los resultados obtenidos, se observó que la conductividad presenta correlaciones directas con seis de las variables que componen el estudio: cloruros, color, DBO, DQO, nitrógeno, grasas y aceites, además del punto o ubicación del sitio de muestreo. Por lo tanto, con la medida de conductividad promedio se puede tener una relación directa con las demás variables contaminantes, que describen la variación de la contaminación en el colector La Doctora, y ello permitirá tomar las medidas de conductividad promedio como medida de la contaminación en el colector La Doctora.

De los resultados arrojados a lo largo de los monitoreos, se observa que en la parte baja de la cuenca se registran incrementos significativos de conductividad, alcanzando máximos de $1017 \mu \mathrm{s} / \mathrm{cm}$. Lo anterior permite deducir que para el DS ARnD se presentan picos altos de concentración de contaminantes, asociados con los vertimientos, lo cual confirma que el incremento de cargas contaminantes en la cuenca se concentra en este distrito. Entre las zonas alta y media, los valores máximos de conductividad variaron entre $161 \mathrm{y}$ $341 \mu \mathrm{s} / \mathrm{cm}$, con mínimos cercanos a los $140 \mu \mathrm{s} / \mathrm{cm}$.

Igualmente, se pudo apreciar un cambio en la naturaleza del AR producido en la zona, con vertidos que son transportados por el colector (desde la zona doméstica a la no doméstica) y que terminan con una presión de contaminantes con responsabilidad del sector industrial, ubicado en la parte baja de la cuenca. Adicionalmente, se observa que a partir de las respuestas de las variables color aparente, color real y cloruros, es posible ver el incremento en el promedio de color aparente entre los tres puntos de muestreo, con valores más altos para la parte baja de la cuenca (valor máximo de 2500 unidades de color), así como incrementos, aunque menos graduales, entre el color real y los cloruros (valores reportados de 116 unidades de color y 71,8 mg/L, respectivamente). 
En las partes alta y media de la cuenca, el promedio de color alcanzó valores de 62 y 75 unidades de color, respectivamente. Lo anterior obedece a que el promedio de color está asociado con las respuestas de la turbiedad del agua, así como con los incrementos observados para la variable, y que se asocian con los grandes volúmenes de agua que son vertidos a la red de alcantarillado.

Finalmente, los cloruros (con valores que alcanzan promedios en la zona baja de la cuenca de $186,9 \mathrm{mg} / \mathrm{L}$ y menores a $50 \mathrm{mg} / \mathrm{L}$ para las otras dos zonas) presentaron una correlación más fuerte con el color real. Lo anterior se debe a los procesos industriales asociados principalmente con la industria textil; en esta, diversas actividades conducen al incremento del valor del $\mathrm{pH}$, y es clara la participación de este tipo de empresas en el área final de la zona estudio (parte baja de la cuenca o distrito sanitario DS ARnD).

\section{Discusión}

Dadas las condiciones de generación de aguas residuales y la calidad o volumen del vertimiento, es posible para el prestador del servicio identificar los puntos más vulnerables de su red de recolección de aguas residuales, pues ello permite identificar potenciales sitios de obstrucción, daño a la red por corrosión o similares; ello podrá, finalmente, incidir en un mejor manejo de las redes y mayor conocimiento para la operación y mantenimiento de estas.

A partir de las condiciones de norma establecidas para el país, es posible identificar las necesidades en términos de nuevas formas de operar o fraccionar los diferentes actores en las cuencas, pues son variables las dinámicas de las poblaciones humanas $\mathrm{y}$, especialmente, de las características de los sectores industriales. Sin embargo, esta diferenciación requiere de un conocimiento muy preciso de los diferentes vertimientos que llegan a una red de aguas residuales (caudales, concentraciones del contaminante, entre otros).

Ahora, con base en la determinación de distritos sanitarios (DS), tal como se ha logrado establecer a lo largo de este artículo, en la cuenca La Doctora del municipio de Sabaneta se logra evidenciar que es factible hacer una diferenciación de distritos a partir de la información de campo y de la distribución espacial de los diferentes actores involucrados: comunidad, servicios o comercios específicos (restaurantes, colegios, barberías, entre otros, e industrias). Es decir, evaluar alternativas de separación de redes de alcantarillado a partir de la calidad del agua residual en las cuencas, lo cual se puede convertir en una acción que permitirá alcanzar dos objetivos. Primero, no realizar mezcla de agua residual
(ARD y ARnD) en las redes de transporte y luego conducirlas a las PTAR dada la mezcla, lo que puede representar mayores costos de tratamiento y podría dificultar su depuración; esta acción ayudará a cumplir las metas planteadas por la ONU en su informe mundial del agua 2017, enfocadas en la separación en la fuente [1] (UNESCO, 2017). Segundo, conducir las ARnD a sitios donde se puedan ofrecer tratamientos a los vertimientos (descentralización del sistema de tratamiento) o remover previamente algunos de sus componentes complejos y que ponen en riesgo el buen desempeño de las PTAR. Esta actividad podrá permitir un tratamiento agrupado y sectorizado de las AR.

Por lo tanto, se espera que se pueda pensar en la generación de alternativas donde se logre de manera conjunta unir esfuerzos de diferentes actores ambientales del territorio en la gestión integral de los vertimientos. Igualmente, explorar asociaciones públicas APP o público privadas APP como las planteadas por [18] (Chen, H., 2017), en las cuales, en asociación con las autoridades y mediante mecanismos como tasas retributivas, se logre crear proyectos de tratamiento de AR industriales sectorizados en las cuencas con distritos sanitarios de ARnD.

Específicamente para la cuenca La Doctora, a partir de los hallazgos planteados en los resultados del presente estudio, se encontró que el tipo $\mathrm{ARnD}$ no está definida realmente por el tipo de sector productivo [13], sino por su caudal. Por esto, para evitar este tipo de situaciones, se propone ahondar en estudios similares y evaluar, de ser posible, la presentación de estudios de caracterización a pequeños vertedores (con la regularidad y rigurosidad que hoy se hace), pues en este caso se encontró que un total de 21.947 puntos de descarga corresponden a usuarios que vierten menos de $14,38 \mathrm{~m}^{3} /$ mes y que son en su mayoría establecimientos comerciales.

A partir de esta investigación, que concentra la gestión de los vertimientos de acuerdo con los DS, se logra definir que tan solo para el DS ARnD hay potencial para ofrecer tratamiento industrial sectorizado para un caudal de $163.835 \mathrm{~m}^{3} / \mathrm{mes}$ (provenientes de 437 industrias).

Igualmente, se destaca la importancia de realizar un mejor uso de la información que arrojan las autodeclaraciones del sector industrial, puesto que esta permitirá llevar a cabo una mejor gestión y operación de la red de alcantarillado. En el presente estudio se evidenció un acatamiento de solo el $11 \%$ de los usuarios en la presentación de las autodeclaraciones de agua residual, lo que permite visualizar la posibilidad u oportunidad de ofrecer alternativas de tratamientos sectorizadas, garantizando el mayor cumplimiento de la 
norma y la formulación de nuevas oportunidades de negocio en el tratamiento y depuración de las AR del sector industrial de la cuenca.

En cuanto al distrito sanitario mixto, este es un distrito que carece de registros significativos, desde el punto de vista de autodeclaraciones, por lo que se recomienda seguir avanzando en procesos de investigación. Con ello se podrán registrar industrias que estén produciendo grandes volúmenes de caudal y que requieran la inclusión de estudios de caracterización. Toda esta apuesta permitirá contar con registros y evaluar el comportamiento de los contaminantes en esta zona especial. También, se recomienda instalar para este distrito (punto en el que se registra la zona de transición del AR en la cuenca, ARD a ARnD) estaciones de calidad móviles y automáticas, con el fin de registrar con más detalle los puntos donde el agua cambia notablemente su composición en términos de los contaminantes vertidos. Esta propuesta se podría fortalecer con sistemas inteligentes de lectura directa y valoración en tiempo real, con lo que se podrá tener mejor gestión del recurso y mayor cuidado y protección de las redes de alcantarillado.

Por último, y como una aplicación especial, se propone evaluar el comportamiento en la aplicación de la tasa retributiva a partir dos miradas diferenciadas. La primera se enfoca en encontrar los puntos donde se deben realizar estudios de caracterización para colectores típicos de ARD, para lo cual se sugiere en el estudio, emplear el punto 1 o la zona definida como la más alta de la cuenca (zona rural). La segunda o la zona mixta se enfoca en la tasa de $\mathrm{ARnD}$, donde se recomienda tomar el punto $3 \mathrm{o}$ la parte más baja de la cuenca (zona influenciada por asentamientos industriales de gran carga contaminante). En ambos estudios se podrá contar con el apoyo de los modelos estadísticos que soportan toda la investigación.

\section{Conclusiones}

Se podría concentrar la población objeto de vigilancia y control en el municipio de Sabaneta en solo 728 instalaciones de un total de 2.914, optimizando los programas de investigación y control de las ARnD en la cuenca. Con esto se disminuye el espectro de usuarios a vigilar en un $75 \%$, dada la variación en volumen vertido y generado entre usuarios (establecimiento comercial versus una gran industria).

Con el estudio se logra verificar que el tipo de actividad productiva de las industrias localizadas en la zona de estudio no tiene necesariamente una relación directa con los contaminantes muestreados, y por lotanto dicha actividad no describe el tipo de contaminación que es vertido a la red, lo cual se deberá determinar de acuerdo con el tamaño de la industria.

Dada la alta correlación encontrada entre las variables cloruros y color en la cuenca, se propone implementar un sistema de localización con el uso de sondas y electrodos (especialmente para cloruros) en puntos estratégicos de la red y monitorear indirectamente el aumento de los niveles de color.

Puesto que el AR ejerce presión en áreas geográficas definidas y diferenciadas, asociadas con el tipo de vertimiento descargado a las redes de alcantarillado, se propone llevar esta propuesta a otras cuencas y delimitar áreas o distritos sanitarios específicos. Para ello, se tendrá en cuenta información histórica, bases de datos e información de calidad y cantidad de AR.

Con base en la determinación de distritos sanitarios se podrán evaluar alternativas de separación en las redes de alcantarillado y plantear tratamientos sectorizados, principalmente para aguas tipo $\mathrm{ARnD}$ en zonas industriales.

En la cuenca La Doctora hay potencial de ofrecer tratamiento industrial sectorizado para un caudal de $163.835 \mathrm{~m}^{3} / \mathrm{mes}$ proveniente de 437 industrias. Lo anterior, gracias al establecimiento de tres distritos sanitarios con tres categorías claramente establecidas: uno, definido como DS ARD en la parte alta; otro denominado DS mixto, localizado en la parte media de la cuenta y un tercero que corresponde a la presión ejercida por ARnD.

Los sistemas de información geográficos (SIG) constituyen un aporte importante para el análisis geoestadístico de las AR, como herramienta de gestión y apoyo en la toma de decisiones operativas y estratégicas, basadas en el análisis espacial de los datos.

\section{Agradecimientos}

Los autores reconocen el apoyo de la Gerencia de Aguas Residuales de EPM y todo el equipo de Investigación y Control, en cabeza del ingeniero León Arturo Yepes Enríquez; además, a Claudia Patricia Martínez Echeverri, ya que sin su apoyo no hubiera sido posible la realización de esta investigación.

\section{Referencias}

[1] UNESCO, "Aguas Residuales: el recurso desaprovechado", Organización de las Naciones Unidas para la Educación, la Ciencia y la Cultura, 2017. 
[2] J. Paavola, "Sewage Pollution and Institutional and Technological Cange in the United States, 1830-1915", Ecological Economics, vol. 69, no. 12, pp. 2517-2524, 2010, doi: 10.1016/j.ecolecon.2010.07.028

[3] S. Tongesayi, T. Tongesayi, "Chapter Seventeen - Water Quality and Public Health: Role of Wastewater", en Chemistry and Water The Science Behind Sustaining the World's Most Crucial Resource, West Long Branch, NJ, USA: El Sevier, 2017, pp. 553596, doi: 10.1016/B978-0-12-809330-6.00017-9

[4] R. K.Sharma, M. Yadav, R. Gupta, "Chapter Five - Water Quality and Sustainability in India: Challenges and Opportunities", en Chemistry and Water The Science Behind Sustaining the World's Most Crucial Resource, West Long Branch, NJ, USA: El Sevier, 2017, pp. 183-205, doi: 10.1016/B978-0-12-809330-6.00005-2

[5] A. S. Tanner, B. S. Mcintosh, D.C.C. Widdowon, M.R. Tillstson, "The water Utility Adoption Model (wUAM): Understanding Influences of organisaional and procedural innovation in a UK water utility", Journal of Cleaner Production, vol. 171, pp. 586-596, 2018, doi: 10.1016/j.jclepro.2016.06.176

[6] Ş. Delipınar, M. Karpuzcu, "Policy, legislative and institutional assessments for integrated river basin management in Turkey", Environmental Science \& Policy., vol 72, pp. 20-29, 2017, doi: 10.1016/j.envsci.2017.02.011

[7] U. When, C. Montalvo, "Exploring the dynamics of water innovation: Foundations for water innovation studies", Journal of Cleaner Production, vol. 171, pp. 119, 2018, doi: 10.1016/j.jclepro.2017.10.118

[8] S. M. Praveenaa, A. Zaharin Arisb, "A baseline study of tropical coastal water quality in Port Dickson, Strait of Malacca", Marine Pollution Bulletin, vol. 67, no. 1-2, pp. 196-199, 2012, doi: 10.1016/j.marpolbul.2012.11.037

[9] X. Wang, J. Zhang, V. Babovic, "Improving real-time forecasting of water quality indicators with combination of process-based models and data assimilation technique", Ecological Indicators., vol. 66, pp. 428-439, 2016, doi: 10.1016/j.ecolind.2016.02.016

[10] C. Granados, E. Sánchez, "Water Reforms, Descentralización and Child Mortality in Colombia, 1990-2005", World Development, vol 53, pp. 68-79, 2014, doi: 10.1016/j.worlddev.2013.01.007
[11] Resolución 0075 de 2011. Por la cual se adopta el formato de reporte sobre el estado de cumplimiento de la norma de vertimiento puntual al alcantarillado público. [En línea]. Disponible en: https://www.minambiente.gov.co/images/GestionIntegr aldelRecursoHidrico/pdf/normativa/Res_0075_de_2011 .pdf.

[12] Decreto 2667 de 2012. Por el cual se reglamenta la tasa retributiva por la utilización directa e indirecta del agua como receptor de vertimientos puntuales, y se toman otras determinaciones. [En línea]. Disponible en: https://www.funcionpublica.gov.co/eva/gestornormativo /norma.php?i=51042.

[13] Resolución 0631 de 2015. Por la cual se establecen los parámetros y valores máximos permisibles en los vertimientos a los cuerpos de agua superficiales y a los sistemas de alcantarillado público. [En línea]: Disponible en:https://www.leyex.info/leyes/Resolucionmads631de2 015.pdf.

[14] Decreto 1076. Por medio del cual se expide el Decreto Único Reglamentario del Sector Ambiente y Desarrollo Sostenible. [En línea]: Disponible en: https://www.funcionpublica.gov.co/eva/gestornormativo /norma_pdf.php?i=78153.

[15] ArcGis Pro, "Obtenido de Análisis de clúster y de valor atípico (I Anselin local de Moran)", ESRI. [En línea]. Disponible en: https://pro.arcgis.com/es/proapp/tool-reference/spatial-statistics/cluster-and-outlieranalysis-anselin-local-moran-s.htm.

[16] E. W. Rice, R. B. Baird, A. D. Eaton, Standard Methods For the Examination of Water and Wastewater, 23nd edition. USA: American Public Health Association, American Water Works Association, Water Environment Federation, 2017.

[17] Protocolo de monitoreo del agua, Instituto de Hidrología, Meteorología y Estudios Ambientales, 2017. [En línea]. Disponible en: http://documentacion.ideam.gov.co/openbiblio/bvirtual/ 023773/protocolo_monitoreo_agua_ideam.pdf

[18] H. Chen, X. Cui, R. Long, T. Yang, D. Zhu, "Application of the public-private partnership model to urban sewage treatment", Journal of Cleaner Production, vol. 142, no. 2, pp. 1065-1074, 2016, doi: 10.1016/j.jclepro.2016.04.152 